\title{
Effects of Ginseng Polysaccharides on Hepatocellular Energy Metabolism in Hepatic Ischemia Reperfusion Injury in Rabbits
}

Haie Chen, Yingchun Ma, Lei Ying, Dan Chen, Yakun Liu, Shan Zhao, Guanlong Li and Wantie Wang*

Department of Pathophysiology, Wenzhou Medical College, Wenzhou, Zhejiang, China

\begin{abstract}
Objective: To investigate the effects of ginseng polysaccharides on hepatocellular energy metabolism in Hepatic Ischemia reperfusion injury of rabbits.

Methods: 30 rabbits were randomly divided into 3 groups: Control Group (C), Ischemia-Reperfusion Injury Group (IR) and Ginseng Polysaccharides Group (GP). We investigated Alanine Aminotransferase (ALT) in plasma Adenosine Triphosphate (ATP), Adenosine Diphosphate (ADP), Adenosine Monphosphate (AMP), Total Adenylic Acid Number (TAN), and Energy Charge (EC) in liver and morphological changes of hepatic cells.

Results: In group IR, the content of ATP and EC in hepatic tissue were obviously less than those of group $\mathrm{C}$ $(P<0.01)$. The content of ADP and AMP was more abundant than those of group $\mathrm{C}(P<0.01)$. In both groups there was little or no significant content of TAN $(P>0.05)$, and was obviously morphological abnormality in hepatic tissue. In group GP, the content of ATP was more abundant $(P<0.01)$ and the content of ADP and AMP were evidently less than those of group IR $(P<0.01$ or $P<0.05)$; The content of ATP was even less than that of group $C(P<0.05)$; the content of TAN had significantly statistical difference between group GP and group $\mathrm{C}(P>0.05)$, and morphological abnormality in hepatic tissue was obviously reduced.
\end{abstract}

Conclusion: Ginseng polysaccharides could attenuate Hepatic Ischemia Reperfusion Injury (HIRI) by improving hepatocellular energy metabolism.

Keywords: Ginseng polysaccharides; Liver; Ischemia reperfusion injury; Energy metabolism

\section{Introduction}

Ginseng polysaccharides are the main effective component of ginseng. Recent years, a large number of studies revealed that it plays a protective role in HIRI [1-3]. Many studies have approved that oxidative stress, microcirculation failure, kinds of cytokines and chemokines, mitochondrial permeability transition, intracellular $\mathrm{Ca}^{2+}$ overloaded and inflammatory cells are relevant to HIRI [4]. The content of plasma ALT is a sensitive index to measure the liver's function, it reflects hepatocellular damage induced by HIRI [5]. ATP is a high-energy phosphate compound, it can achieve energy storage and can release it by converting into ADP, to ensure that the energy is supplied to the cells in the various activities of life. When HIRI happens, ischemia, hypoxia lead to cellular aerobic failure followed by a sharp fall in intracellular ATP synthesis. So, the tissue concentration of ADP and AMP increases [6]. Ginseng polysaccharides can reverse the damage induced by HIRI. We measured the above indexes to study the change of liver's function and energy metabolism when HIRI occurs.

This experiment explained about the rabbit's energy metabolic change in HIRI and effects of ginseng polysaccharides. It is concluded that Ginseng polysaccharides may protect HIRI by changing its energy metabolism and may be of therapeutic benefit for the protection of liver's preoperative period, and can protect HIRI by reducing the decomposition of ATP and improving the production of ATP, so as to delay ATP failure and change its energy metabolism.

\section{Materials and Methods}

\section{Materials}

Thirty specific pathogen-free rabbits with equal number of males and females with a body weight of 2.0-2.4 kg were purchased from Experimental Animal Center of Wenzhou Medical College (Animal
No of WZMC: 220002). The rabbit livers were used in accordance with relevant guidelines and regulations and according to a protocol approved by Animal Care and Use Review Committees of Wenzhou Medical College Health Science Center. The study conforms to the Guide for the Care and Use of Laboratory Animals published by the US National Institutes of Health (NIH Publication No. 85-23, revised 1996).

Ginseng polysaccharides injections ( $2 \mathrm{ml}: 6 \mathrm{mg}$ ) were supplied by Sanxi PuDe pharmaceuticals Co., Ltd., (Batch No of NFDSMB H14023512). Alanine aminotransferase (ALT) monitoring kit was purchased from Nanjing Jiancheng Bioengineering Institute.

\section{Animal model and grouping}

The HIRI model was prepared by anesthetizing the animals with ethyl carbamate $(1.0 \mathrm{~g} / \mathrm{kg}$ body wt) by intravenous administration and restrained in a supine position during the experiments. A tracheal tube was inserted through a tracheostomy. Sterilizing and laparotomizing were performed and the liver hilus was exposed. The blood vessels to the left lateral, median, right median and square lobes were dissociated above the caudate lobes. The vascular clamp was removed after $45 \mathrm{~min}$ and the experiments were continued for $45 \mathrm{~min}$ after the ischemic period [7].

*Corresponding author: Wantie Wang, Professor, Department of Pathophysiology Wenzhou Medical College, Wenzhou, Zhejiang, China, Tel: (86)577-86689817; Fax: (86)577-86689817; E-mail: wwt@wzmc.edu.cn

Received March 06, 2012; Accepted May 23, 2012; Published May 26, 2012

Citation: Chen H, Ma Y, Ying L, Chen D, Liu Y, et al. (2012) Effects of Ginseng Polysaccharides on Hepatocellular Energy Metabolism in Hepatic Ischemia Reperfusion Injury in Rabbits. J Liver 1:107. doi:10.4172/2167-0889.1000107

Copyright: (c) 2012 Chen $\mathrm{H}$, et al. This is an open-access article distributed unde the terms of the Creative Commons Attribution License, which permits unrestricted use, distribution, and reproduction in any medium, provided the original author and source are credited. 
Citation: Chen H, Ma Y, Ying L, Chen D, Liu Y, et al. (2012) Effects of Ginseng Polysaccharides on Hepatocellular Energy Metabolism in Hepatic Ischemia Reperfusion Injury in Rabbits. J Liver 1:107. doi:10.4172/2167-0889.1000107

Page 2 of 4

Animals were divided into 3 groups randomly and each group had the same number of males and females:

(1) Control group (C) $(\mathrm{n}=10)$ : liver hilus was exposed without occluding the blood vessels.

(2) Ischemia Reperfusion group (IR) ( $\mathrm{n}=10)$ : hepatic porta was blocked and reperfused for 45 minutes after 45 min of ischemia.

(3) Ginseng Polysaccharides group (GP) ( $n=10)$ : hepatic porta was blocked and reperfused for $45 \mathrm{~min}$.

$0.3 \mathrm{mg} / \mathrm{kg}$ of Ginseng polysaccharides was injected into external jugular vein for 10 minutes before ischemia. The same volume of normal saline (NS) was injected into group C and group IR. Other operations of group (GP) were the same as group IR. $10 \% \mathrm{KCl}$ was injected iv to kill the animals as each experiment was over.

\section{Detection indexes}

Detection of ALT: At the end of 45 minutes reperfusion, $8 \mathrm{ml}$ of blood from the external jugular venous were collected from three groups, and then kept in dry tubes for testing.

Detection of ATP, ADP, AMP and calculation of TAN, EC: After killing of animals at the end of 45 mins reperfusion, $500 \mathrm{mg}$ of tissues were collected from the left \& middle lobes of liver and homogenised in $5 \mathrm{ml}$ of $\mathrm{HCLO}_{4}(0.6 \mathrm{~mol} / \mathrm{L})$. Tissue lysates were centrifuged at $1200 \mathrm{rpm} / \mathrm{min}$ for $15 \mathrm{mins}$ and the supernatants were added to $1 \mathrm{ml}$ $\mathrm{K}_{2} \mathrm{CO}_{3}(1.2 \mathrm{~mol} / \mathrm{L})$ and again centrifuged for $10 \mathrm{mins}$, then the new supernatants were collected. The contents of ATP, ADP and AMP were determined by High Performance Liquid Chromatography (American Beckman 332 HPLC, chromatographic column: OPSC18). TAN and the wasting of Energy Charge (EC) in energy state were calculated by the following equations:

\section{$\mathrm{TAN}=\mathrm{ATP}+\mathrm{ADP}+\mathrm{AMP}$}

$\mathrm{EC}=(\mathrm{ATP}+0.5 \mathrm{ADP}) / \mathrm{TAN})$.

Sample preparation and observation for electron microscopy: Rabbit's hepatic tissue was taken out quickly at the end of each experiment, washed with icy Normal Saline (N.S) and made into samples in the size of $2 \mathrm{~mm} \times 2 \mathrm{~mm}$. The prepared samples were fixed with $2.5 \%$ Glutaraldehyde and next with $1 \%$ Osmic acid, then embedded specimens were sliced with type LKB- $V$ and were observed under a H-600 transmission electron microscope.

\section{Statistical analysis}

Statistical package SPSS17.0 was used for statistical analysis. All the data was presented as means \pm S.D. (standard deviation) and tests for normality and homoscedasticity were carried out (we did logarithmic conversion with the data in heterogeneity of variance). Intragroup was compared by Dunnett's test. A value of $p<0.05$ was considered significant. Interclass was compared by q test or LSD test. In bivariant correlation test, correlation analysis was deployed by Pearson's Correlation.

\section{Results}

\section{Changes of ALT, ATP, ADP, AMP, TAN and EC in hepatic tissue}

In group IR, the content of ATP in hepatic tissue was obviously less than group $\mathrm{C}(P<0.01)$, EC level was also lower compared with group $\mathrm{C}(\mathrm{p}<0.01), \mathrm{ALT}$ in plasma and the contents of ADP and AMP were more abundant than group $\mathrm{C}(P<0.01)$, and in both groups there was no significant difference within the content of TAN $(P>0.05)$, and morphological abnormality existed in hepatic tissues of group IR. In group GP, the content of ATP was more abundant than group IR $(P<0.01)$ and EC level had the same tendency $(\mathrm{p}<0.01)$, ALT in plasma, the contents of ADP and AMP were evidently less than those of group IR $(P<0.01$ or $P<0.05)$; The content of ATP was even less than group $\mathrm{C}$ $(P<0.05)$; the content of TAN had no significant statistical difference between group GP and group C $(P>0.05)$, and morphological abnormality in hepatic tissue was obviously reduced (Table 1).

\section{The relationship between all indexes of hepatic tissue and} ALT in plasma at the end of 45 min of reperfusion

The contents of ALT, ATP, ADP and AMP are shown in four graphs (Figure 1,2,3,4, $\mathrm{n}=30$ ). Linear correlation analysis showed that, at the end of $45 \mathrm{~min}$, ATP is in negative correlation with ALT in hepatic tissue, the correlation coefficient $\mathrm{r}$ is $-0.629, P<0.01$; ADP, AMP and ALT were in positive correlation, the correlation coefficients $r$ were -0.685 and -0.779 respectively at $P<0.01$ (Table 2 ).

\section{Ultrastructural changes in hepatic tissue}

The structure of hepatic tissue in group $\mathrm{C}$ was normal; In group IR, there were swelled hepatic cells, few microvillus in bile capillaries cavity, expanded Disiens gap, a large number of expanded rough endoplasmic reticulum in hepatic cells, expanded caryotheca gap, evidently expanded bile capillaries and very few siltation phenomenon of bile; in group GP, there were existing swelling of local hepatic cells, more superfacial microvillus, light expanded Disiens gap, slight widen rough endoplasmic reticulum, mildly swelled chondriome, few of which vacuolized, slight expanding of bile capillaries, no significant decreases of microvillus in bile capillaries cavity (Figure 5).

\section{Discussion}

Liver is the largest parenchymatous organ in human body and is rich in circulating blood. It is sensitive to ischemia and hypoxia due to its poor ability to tolerate them [8,9]. Especially sinusoidal endotheliocytes are more easily influenced by HIRI and are accompanied with necrosis. Kawarnura and the like have proved that in hepatic ischemic phase, hypoxic metabolism resulting in lack of energy is one of the most important causes that induce hepatic tissue injury after ischemia reperfusion. The disturbance of energy metabolism in the state of ischemia and hypoxia is the initial factor leading to hepatic pathophysiological changes in the process of HIRI [10].

In ischemia reperfusion, ischemia and hypoxia lead to the disturbance of cellular aerobic metabolism which makes intracellular

\begin{tabular}{|c|c|c|c|c|c|}
\hline Group & ALT & ATP & ADP & AMP & TAN \\
\hline group C & $36.4 \pm 2.99$ & $3.42 \pm 0.75$ & $1.16 \pm 0.29$ & $1.39 \pm 0.25$ & $5.98 \pm 0.49$ \\
\hline group IR & $58.9 \pm 6.31^{\#}$ & $0.98 \pm 0.25^{\#}$ & $2.33 \pm 0.54^{\# \#}$ & $3.32 \pm 1.02^{\#}$ & $6.49 \pm 1.4$ \\
\hline group GP & $45.2 \pm 7.33^{\mathbf{\Delta}}$ & $2.80 \pm 0.77^{\mathbf{\Delta}} \#$ & $1.83 \pm 0.38^{\mathbf{4} \#}$ & $1.50 \pm 0.23^{\mathbf{\Delta}}$ & $6.34 \pm 0.05^{\# \#}$ \\
\hline
\end{tabular}

Compared with group $\mathrm{C}: \# P<0.05, \# \#<0.01$; Compared with group IR: $\boldsymbol{\Delta} P<0.05, \boldsymbol{\Delta} \mathbf{\Delta} P<0.01$.

Table 1: The content of ALT, ATP, ADP, AMP, TAN \& EC in hepatic tissue of different groups. 
Citation: Chen H, Ma Y, Ying L, Chen D, Liu Y, et al. (2012) Effects of Ginseng Polysaccharides on Hepatocellular Energy Metabolism in Hepatic Ischemia Reperfusion Injury in Rabbits. J Liver 1:107. doi:10.4172/2167-0889.1000107
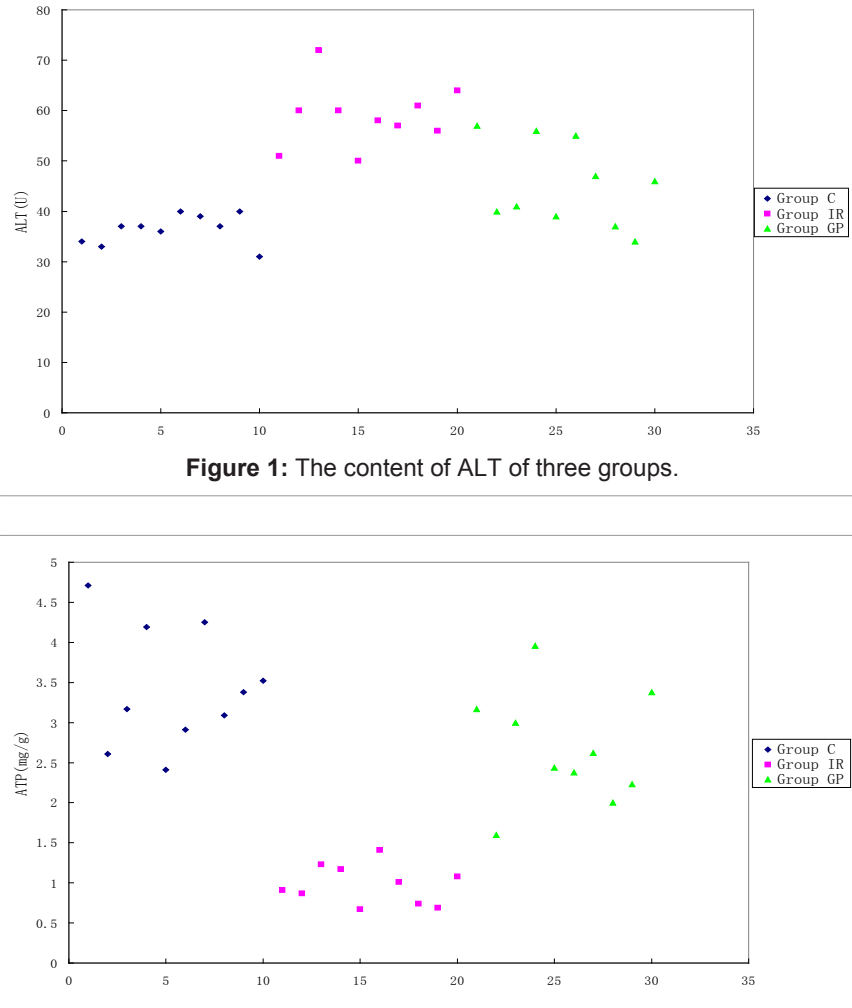

Figure 2: The content of ATP of three groups.

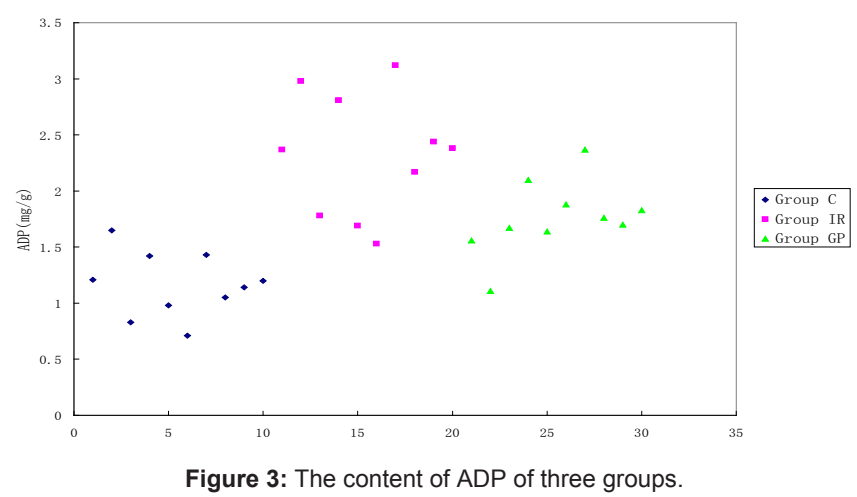

synthesis of ATP to be sharply reduced. Energy deficiency induces permeability of cell membrane. Meanwhile, the function of $\mathrm{Na}^{+}-\mathrm{K}^{+}-$ ATPase on cell membrane is reduced, and inflow of $\mathrm{Na}^{+}$and efflux of $\mathrm{K}^{+}$cause rapid cellular edema. In the late phase of cellular hypoxic injury, the inflow of extracellular $\mathrm{Ca}^{2+}$ increases and $\mathrm{Ca}^{2+}$ accumulates in hepatic cells. Simultaneously, as a result of reduced synthesis of ATP, the activity of $\mathrm{Ca}^{2+}$-ATPase is depressed and the exclusion of intracellular $\mathrm{Ca}^{2+}$ decreases, intracellular $\mathrm{Ca}^{2+}$ overcharges $[9,11,12]$. In hypoxia, intracellular and extracellular calcium paradox is the biochemical base of organ injury in ischemia reperfusion. When liver is in the state of ischemia, the content of ATP in hepatic cells is low, edema exists in hepatic cells. Hypoxia can also cause the activation of proteinase, the skeleton structure of hepatic cells are destroyed. Hepatic sinusoids become narrow. Meanwhile, hepatic edema occurs, endothelial cells swell to death, and intravascular microthrombus forms and blocks hepatic microcirculation [13].
In recent years a series of studies have proved that Ginseng polysaccharides can prevent liver injury induced by carbon tetrachloride through the Down regulation of oxidative stress and inflammatory response [14]. It can also protect mitochondria by inhibiting mitochondrial swelling and improving energy metabolism. It has the pharmaceutical activities of antihypoxia, antioxidation and improving energy status [15]. Mitochondria are the main place of energy metabolism and most of these are located in liver, so liver is the most important place of energy metabolism. Our previous studies have proved that Ginseng Polysaccharides can reduce HIRI by reducing the activity of $\mathrm{OX}$, suppressing the production oxygen-derived free radicals, toning up antioxidase like SOD, GSG-Px and suppressing lipid peroxidization [16]. Ginseng polysaccharides can balance NO/ET to reduce HIRI [17].

This experiment revealed that when ischemia reperfusion happened in liver, the content of ATP and EC obviously declined, but the content of ADP and AMP apparently raised, ALT in plasma also raised. Simultaneously, abnormality of hepatic function, hepatic cellular morphology and structure happened via GP pretreatment, the content of ATP and EC level raised, the content of ADP and AMP declined, ALT

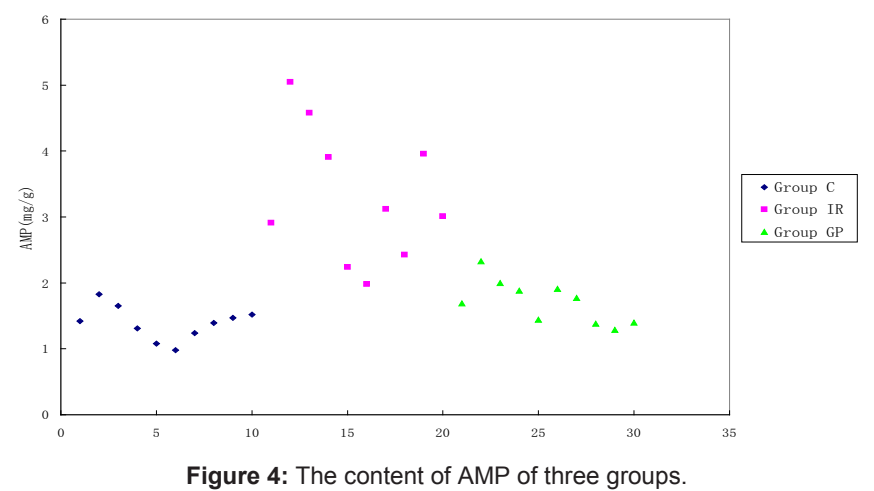

\begin{tabular}{|c|c|c|}
\hline Parameters & $r$ & $p$ \\
\hline ALT & & \\
\hline ATP & -0.629 & $\mathrm{P}<0.01$ \\
\hline ADP & 0.685 & $\mathrm{P}<0.01$ \\
\hline AMP & 0.779 & $\mathrm{P}<0.01$ \\
\hline
\end{tabular}

Table 2: Correlations between All Indexes Of Hepatic Tissue And ALT In Plasma

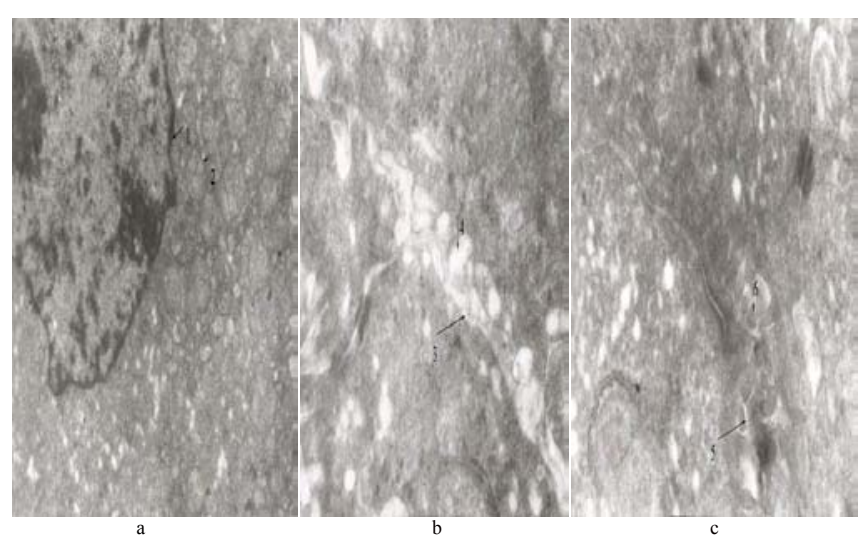

Figure 5: The ultrastructure of hepatic tissue under electron microscope of the three groups. TEM $\times 25 \mathrm{~K} 5 \mathrm{a}$ : group C; $5 \mathrm{~b}$ : group IR; 5c: group GP (1 nucleus 2 mitochondria 3,5 bile capillary 4,6 microvillus). 
Citation: Chen H, Ma Y, Ying L, Chen D, Liu Y, et al. (2012) Effects of Ginseng Polysaccharides on Hepatocellular Energy Metabolism in Hepatic Ischemia Reperfusion Injury in Rabbits. J Liver 1:107. doi:10.4172/2167-0889.1000107

had the same variation tendency and functional injury of hepatic cells reduced, and morphological abnormal changes in hepatic tissue also obviously decreased. Combined with the above graphs (Figures 1-4) and data (Table 2), we can conclude that hepatic ATP was significantly negatively correlated with plasmatic ALT $(\mathrm{P}<0.01)$, and ADP and AMP were significantly positively correlated with plasmatic ALT $(\mathrm{P}<0.01)$, which indicated that lack of energy-rich phosphate compound might be one of the main factors leading to liver's dysfunction induced by HIRI. All the above can proved that ginseng polysaccharides can alleviate HIRI by increasing hepatic metabolism. The mechanism may be that, ginseng polysaccharides can reduce the decomposition of ATP and improve the production of ATP, so as to delay ATP failure, which can raise energy reserve for hepatic cells, improve hypoxic conditions in hepatic tissue, and cellular edema, cellular $\mathrm{Ca}^{2+}$ overloaded and hepatic microcirculatory disturbance from occurring, so as to remove both biochemical fundament and pathological fundament of the formation of ischemia reperfusion injury in liver, also prevent and treat it.

\section{Acknowledgements}

Supported by science and technology bureau of Wenzhou (Y20060195).

\section{References}

1. Chen Di-long, Liu Yong-gang, Wang Ya-ping, Chen Ting-mei, Zheng Min, et al (2005) Effects of Ginseng polysaccharide on proliferation and differentiation of K562 cells. Acta Academiae Medicine Militaris Tertiae 27: 517-520.

2. Zhang Bin, Lin Rui-chao, Feng Fang (2004) Research survey on Ginseng polysaccharides. Chinese Pharmaceutical Affairs 18: 566-569.

3. Wu Rong, Gao Shou-hong, Xin Hai-liang (2007) Research survey on Ginseng against damage. Journal of pharmacal practice 25: 364-366.

4. Teoh NC, Farrell GC (2003) Hepatic ischemia reperfusion injury: pathogenic mechanisms and basis for hepatoprotection. J Gastroenterol Hepatol 18: 891902.

5. Nishimura T, Yoshida Y, Watanabe F, Koseki M, Nishida T, et.al (1986) Blood level of mitochondrial aspartate aminotransferase as an indicator of the extent of ischemic necrosis of the rat liver. Hepatology 6: 701-707.

6. Sener G, Sehirli O, Ercan F, Sirvanci S, Gedik N, et al. (2005) Protective effect of MESNA (2-Mercaptoethane Sulfonate) against hepatic ischemia/ reperfusion injury in rats. Surg Today 35: 575-580.

7. Hasselgren PO, Bilber B, Fornander J (1983) Improved blood flow and protein synthesis in the postischemic liver following infusion of dopamine. J Surg Res 34: 44-52.

8. Zhang Hong-yi, Li Jie-lei (2000) Actuality and progress of liver transplantation. Journal of general hospital of air force 16: 29.

9. Liu X, Liu Z, Shi J (2000) Experimental study on the effects of radix Ginseng rubra on cardiomyocyte apoptosis after ischemia and reperfusion in rats. Chinese Journal of histochemistry and cytochemistry 9: 261-263.

10. Wang Chenyou, Ni Yong, Li Weiping, He Hai-ping, Wang Jian-zhong (2001) The chance of hepatic tissue $\mathrm{PO} 2, \mathrm{PCO} 2, \mathrm{PH}$ and temperature in ischemia reperfusion injury in rabbit liver. Journal of Hepatobiliary Surgery 9: 229-230.

11. Tang Li-jun,Tian Fu-zhou (2003) Research actuality of low-temperature holding-reperfusion injury of alohisto transplanted liver. Journal of Military Surgeon in Southwest China 5: 27-29.

12. Wang Da-yong, Zhang Jin-zhe, Qiu Xiao-hong, Wang Yan-xia (2003) An experimental study of hepatic ischemia-reperfusion injury. Chinese Journal of Pediatric Surgery 24: 534-536.

13. Wang Chenyou, Ni Yong, Liu Zuo, Zheng Shusen (2002) Experimental study of injury and it's mechanism in the ischemia and reperfusion period in rabbit liver. Journal of Hepatobiliary Surgery 10: 470-472.

14. Li XT, Chen R, Jin LM, Chen HY (2009) Regulation on energy metabolism and protection on mitochondria of Panax ginseng polysaccharide. Am J Chin Med 37: 1139-1152.

15. Ji-Young Shim, Mi-Hyoung Kim, Hyung-Doo Kim, Ji-Yeon Ahn, Yeon-Sook Yun, et al. (2010) Protective action of the immunomodulator ginsan against carbon tetrachloride-induced liver injury via control of oxidative stress and inflammatory response. Toxicol Appl Pharmacol 242: 318-325.

16. Wantie Wang, Lu Shi, Yingqi Liang, Xuguang Jia, Liang Chen, et al. (2009) Ginseng polysaurides interfering changes of lipid peroxidation during hepatic ischemia reperfusion in rabbits. Chinese Journal of Arteriosclerosis 17: 109112

17. Yingqi Liang, Yanrong Zheng, Wantie Wang (2009) Effects of ginseng polysauharides on NO, ET and ALT in hepatic ischemia reperfusion of rabbits. Journal of Heptopancreatobiliary Surgery 21:24-26, 33. 